Original article

\title{
Diet quality in early and mid-childhood in relation to trajectories of growth and body composition
}

\author{
Anh N. Nguyen ${ }^{\mathrm{a}, \mathrm{b}}$, Vincent Jen ${ }^{\mathrm{a}}$, Vincent W.V. Jaddoe ${ }^{\mathrm{a}, \mathrm{b}, \mathrm{c}}$, Fernando Rivadeneira ${ }^{\mathrm{d}}$, \\ Pauline W. Jansen ${ }^{\text {e, f }}$, M. Arfan Ikram a, Trudy Voortman ${ }^{\text {a, * }}$ \\ a Department of Epidemiology, Erasmus University Medical Center, PO Box 2040, 3000 CA, Rotterdam, the Netherlands \\ ${ }^{\mathrm{b}}$ The Generation R Study Group, Erasmus University Medical Center, PO Box 2040, 3000 CA, Rotterdam, the Netherlands

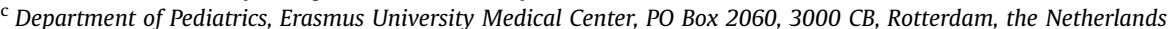 \\ ${ }^{\mathrm{d}}$ Department of Internal Medicine, Erasmus University Medical Center, PO Box 2040, 3000 CA, Rotterdam, the Netherlands \\ e Department of Child and Adolescent Psychiatry/Psychology, Erasmus University Medical Center, PO Box 2060, 3000 CB, Rotterdam, the Netherlands \\ ${ }^{\mathrm{f}}$ Department of Psychology, Education and Child Studies, Erasmus University Rotterdam, PO Box 1738, 3000 DR, Rotterdam, the Netherlands
}

\section{A R T I C L E I N F O}

\section{Article history:}

Received 25 October 2018

Accepted 14 March 2019

\section{Keywords:}

Diet quality

Growth

Body composition

Children

Cohort

\begin{abstract}
S U M M A R Y
Background: A balanced diet in childhood is important for growth and development. We aimed to examine associations of overall diet quality in both early and mid-childhood with trajectories of growth and body composition until age 10 years.

Methods: We included 3991 children from the Generation R Study, a population-based, prospective cohort in Rotterdam, the Netherlands. At child's ages of 1 and 8 years, dietary intake was assessed using food-frequency questionnaires to calculate diet quality scores (0-10), which measure adherence to agespecific dietary guidelines. Height and weight were measured repeatedly between ages 1 and 10 years. Body composition was assessed using dual-energy X-ray absorptiometry at ages 6 and 10 years. We calculated sex- and age-specific SD-scores for body mass index (BMI), fat mass index (FMI), fat-free mass index (FFMI), and body fat percentage (BF\%).

Results: After adjustment for socioeconomic and lifestyle factors, results from linear mixed models showed that higher diet quality at 1 year was associated with higher height, weight, and BMI up to age 10 years. Using linear regression analyses, similar associations were observed for diet quality at 8 years. For diet quality at both time points, positive associations with BMI were fully driven by a higher FFMI ( $\beta=0.07$ SDS, 95\%CI: $0.05,0.10$ for diet quality at 8 years), and not FMI or BF\%. Most of the observed associations were independent of diet quality at the other time point.

Conclusion: We observed that better diet quality in both early and mid-childhood was associated with higher height, weight, and FFMI, but not with body fatness up to age 10 years. This was independent of diet quality at an earlier or later time point. Our findings suggest that dietary intake according to dietary guidelines may have a beneficial impact on growth and body composition throughout childhood.
\end{abstract}

(c) 2019 Elsevier Ltd and European Society for Clinical Nutrition and Metabolism. All rights reserved.

\section{Introduction}

Nutrition in childhood is important for growth and development of the child, and for health later in life [1]. Previous studies

Abbreviations: BMI, Body mass index; CI, Confidence interval; DXA, Dual-energy X-ray absorptiometry; FFO, Food-frequency questionnaire; FMI, Fat mass index; FFMI, Fat-free mass index; IQR, Interquartile range; SDS, Standard deviation score.

* Corresponding author. Department of Epidemiology, Erasmus MC Office Na2716, PO Box 2040, 3000 CA, Rotterdam, the Netherlands. Fax: +31 107044657

E-mail address: trudy.voortman@erasmusmc.nl (T. Voortman). reported that dietary intake of certain nutrients or foods, such as protein, dietary fat, or sugar-sweetened beverages, are associated with children's obesity risk and body composition [2-6]. Childhood obesity may cause serious health complications, and may increase the risk of obesity in adulthood [7] and thereby the risk of coronary heart diseases, diabetes, and premature death [8].

Children consume a variety of foods rather than single nutrients and foods, and these different nutrients and foods interact [9]. Studying overall dietary patterns takes these interactions into account and may be more applicable in public health practices. Dietary patterns can either be data-driven (i.e., based on the variation 
of dietary intake data within a study population) or predefined (e.g., based on specific dietary guidelines or recommendations) [9]. A review including seven studies among children showed positive associations of data-driven dietary patterns characterized by intakes of energy-dense, high-fat, and low-fiber foods with later obesity risk [10]. However, most studies only examined body mass index (BMI) as a measure of obesity, and only one of the cohorts included in this review used dual-energy X-ray absorptiometry (DXA) to assess body fat mass, but not fat-free mass [11]. In addition, a Canadian study observed that children aged 8-10 years with a higher score on a predefined diet quality index gained less body fat over a 2-year period [12]. In contrast to these studies in schoolage children, we previously observed in the Generation R Study that a higher predefined diet quality score at age 1 year was not associated with fat mass at age 6 years, but rather with a higher fatfree mass [13]. However, whether these associations track into later childhood and whether diet in early and mid-childhood differently affects body composition remains unclear.

Therefore, we aimed to first extend our previous analyses on diet quality at age 1 year in relation to body composition at age 6 years [13] with data on growth and detailed measures of body composition up to age 10 years, taking into account diet quality in mid-childhood. As a second aim, we explored associations of overall diet quality at age 8 years with anthropometrics and body composition at age 10 years. For both aims, we examined whether associations are independent of diet quality at the other time point.

\section{Methods}

\subsection{Study design and population}

This study was embedded within the Generation R Study, an ongoing population-based prospective cohort from fetal life onward in the Netherlands [14]. Pregnant women were enrolled between April 2002 and January 2006 and a total of 9749 live-born children were available for follow-up. Parents of all participating children provided written informed consent and approval was obtained from the medical ethical committee of Erasmus University Medical Center, Rotterdam [14].

At the child's age of 1 year, a food-frequency questionnaire (FFQ) to assess diet in early childhood was sent to parents of 5088 children. Dietary data was available for 3629 of the children [15]. Of these children, 3573 had data on anthropometrics and 3122 on body composition available at one or more time points up to age 10 years. At the age of 8 years, an FFQ was sent to parents of 7662 children to assess mid-childhood diet. Data on dietary intake was available for 4733 of these children [16]. Around the age of 10 years, we had data available on anthropometrics for 3991 children and on body composition for 3950 children (Fig. 1).

\subsection{Measures}

\subsubsection{Diet quality in early childhood}

As previously described in detail [15,17], dietary intake in early childhood was assessed at a median age of 12.9 months (interquartile range (IQR) 12.7-14.0) with a semi-quantitative FFQ covering the past month. Energy and nutrient intakes were calculated using the Dutch Food Composition Table. The FFQ was validated against three 24 -h recalls in 32 Dutch children, which showed reasonable to good intraclass correlation coefficients for nutrient intake ranging from 0.4 to 0.7 [15]. We applied a previously defined diet quality score for preschool children, which was constructed based on age-specific dietary guidelines [15]. The ten following components were included: intake of vegetables ( $\geq 100 \mathrm{~g} / \mathrm{d})$; fruit $(\geq 150 \mathrm{~g} / \mathrm{d})$; bread and cereals $(\geq 70 \mathrm{~g} / \mathrm{d})$; rice, pasta, potatoes, and legumes ( $\geq 70 \mathrm{~g} / \mathrm{d})$; dairy $(\geq 350 \mathrm{~g} / \mathrm{d})$; meat, poultry, eggs, and meat substitutes ( $\geq 35 \mathrm{~g} / \mathrm{d})$; fish ( $\geq 15 \mathrm{~g} /$ d); oils and fats ( $\geq 25 \mathrm{~g} / \mathrm{d})$; candy and snacks ( $\leq 20 \mathrm{~g} / \mathrm{d})$; and sugar-sweetened beverages ( $\leq 100 \mathrm{~g} / \mathrm{d})$ [15]. For each component, ratios of reported intakes and recommended intakes were calculated, capped at 1 . For example; a vegetable intake of $60 \mathrm{~g} /$ $\mathrm{d}$ resulted in a score of 0.6 (60 divided by 100) for the vegetable component. The scores were reversely coded for the "candy and snacks' and 'sugar-sweetened beverages' components, meaning that higher scores reflected lower intakes. Scores for the individual components (ranging from 0 to 1 ) were summed, resulting in an overall score between 0 and 10, with higher scores representing a healthier diet [15]. Previous evaluation of this diet score in the Generation R cohort showed adequate construct validity; it was positively associated with intakes of nutrients considered healthy and inversely associated with intakes of unhealthy nutrients [15].

\subsubsection{Diet quality in mid-childhood}

Dietary intake in mid-childhood was assessed at a median age of 8.1 years (IQR 8.0-8.2) with a semi-quantitative FFQ covering the past month, as described in detail elsewhere $[16,18]$. Energy and nutrient intakes were calculated using the Dutch Food Composition Table. The FFQ was validated for energy intake against energy expenditure measured with the doubly labeled water method. This validation showed good correlation (Pearson's $r=0.6$ ) and BlandAltman mean-difference plots showed no relevant systematic bias [18]. We applied a previously defined diet quality score for schoolage children reflecting adherence to age-specific dietary guidelines [16]. This score included the following ten components: intake of fruit ( $\geq 150 \mathrm{~g} / \mathrm{d}$ ); vegetables ( $\geq 150 \mathrm{~g} / \mathrm{d}$ ); whole grains ( $\geq 90 \mathrm{~g} / \mathrm{d})$; fish ( $\geq 60 \mathrm{~g} / \mathrm{w}$ ); legumes ( $\geq 84 \mathrm{~g} / \mathrm{w})$; nuts ( $\geq 15 \mathrm{~g} / \mathrm{d})$; dairy ( $\geq 300 \mathrm{~g} /$ d); oils and fats ( $\geq 30 \mathrm{~g} / \mathrm{d})$; sugar-containing beverages $(\leq 150 \mathrm{~g} / \mathrm{d})$; and meat $(\leq 250 \mathrm{~g} / \mathrm{w})$. Similar to the approach used for the diet score for preschool children, ratios of reported intakes and recommended intakes were calculated for each component, with reverse coding for the 'sugar-containing beverages' and 'meat' components. The component scores were summed into an overall diet quality score (0-10). Further details on the diet score and its construct validity are reported elsewhere [16].

\subsubsection{Anthropometrics and body composition}

Children's anthropometrics were measured at eight different time points between ages 1 year and 10 years. All measurements were performed without shoes and heavy clothing. Up to age 4 years, measurements were taken during routine visits at the Child Health Centers at median ages of 14.3 (IQR 14.1-14.6), 18.3 (IQR 18.1-18.9), 24.7 (IQR 24.2-25.6), 30.5 (IQR 30.1-31.3), 36.6 (IQR 36.2-37.4), and 45.8 (IQR 45.3-46.6) months. At the ages of 6.0 (IQR 5.8-6.2) and 9.7 (IQR 9.6-9.8) years, measurements were performed during visits to our research center at Erasmus Medical Center. Weight was measured with a mechanical personal scale (SECA, Almere, the Netherlands), and height was measured with a Harpenden stadiometer (Holtain Limited, DYFED, U.K.). During these visits, we also measured body composition (fat and lean mass) with a DXA scanner (iDXA, Ge-Lunar, 2008, Madison, WI, USA) using enCORE software version 13.6. We calculated BMI (weight $(\mathrm{kg}) /$ height $(\mathrm{m})^{2}$ ), fat mass index (FMI) (fat mass $(\mathrm{kg}) /$ height $\left.(\mathrm{m})^{2}\right)$, fat-free mass index (FFMI) (fat-free mass $(\mathrm{kg}) /$ height $\left.(\mathrm{m})^{2}\right)$, and body fat percentage $(\mathrm{BF} \%$ ) (fat mass as percentage of total body weight). Overweight status was defined based according to Cole's criteria [19]. Subsequently, we calculated age- and sex-specific standard deviation scores (SDS) for all outcomes based on available data from participants in the Generation R Study [14]. 


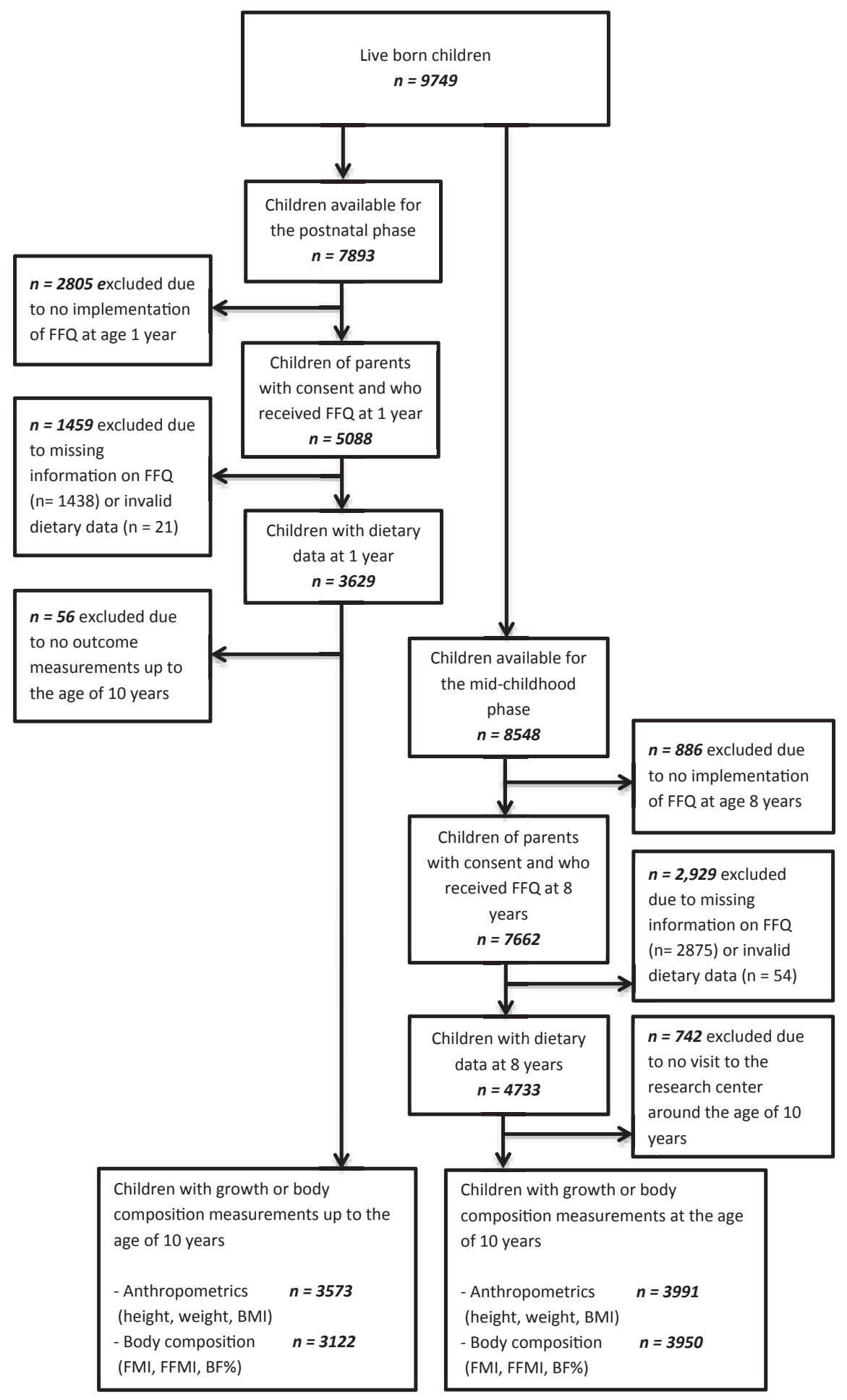

Fig. 1. Flowchart of population for analysis.

\subsubsection{Covariates}

We assessed several socioeconomic and lifestyle factors at study enrollment, in infancy, or in childhood. Information on maternal age, maternal educational level (low; high), parity (nulliparous; multiparous), folic acid supplement use in early pregnancy (no; started in first ten weeks; started periconceptional), and household income $(<2200$; $\geq 2200$ Euros/month) was obtained using questionnaires at enrollment in the study. During each trimester, questionnaires were used to assess whether mothers drank alcohol (never; until pregnancy was known; continued drinking occasionally; continued drinking frequently) and smoked (never; until pregnancy was known; continued smoking during pregnancy). Maternal height and weight were measured at our research center at enrollment, and BMI was calculated.

Information on child's date of birth and sex was obtained from medical records. Child's ethnicity (Dutch; non-Dutch) was defined based on the country of birth of the parents, on which information was obtained with questionnaires. Information on breastfeeding 
was obtained for the first 4 months of life (never; partially; exclusively) via questionnaires.

Around age 10 years, we used questionnaires to obtain information on child's participation in sport activities $(<2 ; \geq 2 \mathrm{~h} /$ week) and screen time, defined as time watching television and/or using computers ( $<2 ; \geq 2 \mathrm{~h} /$ day). Questionnaires were also used to update information on maternal smoking status (never; former; current) and household income $(<2800 ; \geq 2800$ Euros/month). In addition, mother's height and weight were measured to update their BMI.

\subsection{Statistical analyses}

For our first aim, linear mixed models were used to examine associations of diet quality at age 1 year with trajectories of growth between ages 1 and 10 years and body composition between ages 6 and 10 years. This method incorporates all available repeated measurements of the outcomes simultaneously and takes into account that these measurements are correlated within participants. We used likelihood ratio tests to determine a suitable fixed-effect structure and a random effect structure, which we used in each of the longitudinal models. The fixed effect structure was specified using three multivariable models and the random effects structure included a random intercept for the body composition outcomes and a random intercept and slope for time of repeated outcome measures for the growth outcomes. Covariates were selected based on previous literature or a change of $\geq 10 \%$ in effect estimates when they were entered stepwise in model 1 . Model 1 included child's sex, ethnicity, age at dietary assessment, and total energy intake. The second model was additionally adjusted for several socioeconomic and lifestyle factors: maternal age, maternal educational level, parity, folic acid supplement use, household income, alcohol intake during pregnancy, smoking during pregnancy, breastfeeding, sports, and screen time. To examine whether associations of diet quality in early childhood with trajectories of growth and body composition were independent of diet in mid-childhood, model 3 was additionally adjusted for diet quality at the age of 8 years. To examine whether diet quality modified trajectories of growth and body composition, we included interactions between diet quality and age of outcome measures in the fixed effects structure. To examine whether associations of diet quality with growth and body composition differed by child's sex, an interaction term was included in the models.

For our second aim, we used linear regression models to analyze associations of diet quality at age 8 years with child's anthropometrics and body composition at age 10 years. These associations were analyzed using the previously mentioned models 1,2 , and 3, with some adaptations in models 2 and 3 . In model 2, the early-life factors were replaced by factors that were more relevant in later childhood (e.g., smoking during pregnancy was replaced by maternal smoking status at the 10 -year visit). To examine whether associations were independent of early-childhood dietary factors, model 3 was additionally adjusted for diet quality in early childhood and breastfeeding.

Because the FFQs were originally developed for Dutch populations, we performed sensitivity analyses restricted to participants with a Dutch ethnic background. To verify that associations of the diet scores were not driven by one specific component of the score, we repeated the analyses excluding each component at a time (i.e., diet score including nine components instead of ten). To reduce the possibility of reverse causation, analyses were repeated excluding children with overweight or obesity at baseline. For the linear mixed models, we also performed sensitivity analyses in which we excluded outcome measurements that were taken during the first year following dietary assessment to examine whether these measures drive or attenuate the associations. To reduce potential bias due to missing values on covariates (ranging from $0 \%$ to $28.1 \%$ ), these variables were multiple imputed ( $n=10$ imputations) [20]. Exposures and outcomes were not imputed. When the diet quality score was included as a confounder in the analyses, the multiple-imputed variable was used; when it was used as the main exposure, the unimputed values were used. We present pooled regression coefficients of the 10 imputed datasets. Results were considered statistically significant at $P<0.05$, two-sided alpha error. The statistical analyses were carried out using SPSS statistics version 21.0 (IBM Inc., Armonk, NY, USA) and R version 3.4.1 (The R Foundation for Statistical Computing, Vienna, Austria).

\section{Results}

\subsection{Population characteristics}

Because we observed significant interactions of diet quality with sex in some of the analyses, Table 1 presents characteristics of the total study population and stratified by sex. The majority of the children had a Dutch ethnic background (67.4\%), came from households with a higher income (69.5\%), or played sports for more than two hours/week (66.4\%). Mean $( \pm S D)$ diet quality of the children was $4.3( \pm 1.3)$ out of a maximum score of 10 at age 1 year and $4.6( \pm 1.2)$ at age 8 years, indicating that adherence to dietary guidelines at both time points was suboptimal $[15,16]$. Although boys had a slightly higher diet quality score than girls at both ages (Table 1), there was no difference after adjustment for total energy intake $[15,16]$. Diet quality at the two time points was positively but weakly correlated $(\mathrm{r}=0.2, \mathrm{p}<0.01)[16]$.

\subsection{Diet quality in early childhood}

One point higher diet quality score at the age of 1 year was associated with a 0.05 SDS greater height $(95 \% \mathrm{CI}: 0.02,0.08)$ and a 0.06 SDS higher weight $(95 \% \mathrm{CI}: 0.04,0.09)$ up to the age of 10 years (model 2, Table 2). Additional adjustment for diet quality in midchildhood hardly affected these associations (model 3, Table 2). Also, we observed a positive association between diet quality at age 1 year and BMI up to age 10 years ( $\beta=0.05$ SDS, 95\%CI: $0.02,0.08$ ), which was completely driven by a higher FFMI $(\beta=0.04,95 \% \mathrm{CI}$ : $0.004,0.07$ ), and not FMI or BF\% (model 2, Table 2). The direction of the associations remained similar after additional adjustment for diet quality at age 8 years, but the association with FFMI was no longer statistically significant $(\beta=0.03,95 \% \mathrm{CI}$ : $-0.01,0.06)$ (model 3 , Table 2). These associations did not differ between boys and girls (p-for-interaction $>0.05$ for all outcomes).

\subsection{Diet quality in mid-childhood}

For our analyses on diet in mid-childhood, we observed that also higher diet quality score at the age of 8 years was associated with greater height ( $\beta=0.06$ SDS per one point higher diet score, $95 \% \mathrm{CI}$ : $0.03,0.08)$ and higher weight $(\beta=0.04,95 \% \mathrm{CI}: 0.02,0.07)$ at the age of 10 years (model 2, Table 3 ). These associations attenuated slightly, but remained statistically significant after additional adjustment for early-childhood diet (model 3, Table 3). We also observed a positive association with BMI at age 10 years $(\beta=0.03$, $95 \% \mathrm{Cl}: 0.003,0.05)$ (model 2 , Table 3 ), but this association was no longer statistically significant after additional adjustment for diet in early childhood $(\beta=0.02,95 \% \mathrm{CI}$ : $-0.003,0.04)$ (model 3, Table 3 ). When we further examined fat mass and fat-free mass, we observed an association between a higher diet quality at 8 years and a higher FFMI ( $\beta=0.07,95 \% \mathrm{CI}: 0.05,0.10)$, but not FMI or $\mathrm{BF} \%$ (model 2, Table 3). This association with FFMI remained similar 
Table 1

Characteristics of the population for analysis.

\begin{tabular}{|c|c|c|c|}
\hline & \multicolumn{3}{|l|}{ Mean \pm SD, median (IQR), or \% } \\
\hline & Total population $(n=3991)$ & Girls $(n=2022)$ & Boys $(n=1969)$ \\
\hline \multicolumn{4}{|l|}{ Child characteristics } \\
\hline \multicolumn{4}{|l|}{ Sex } \\
\hline Girls & $50.7 \%$ & - & - \\
\hline \multicolumn{4}{|l|}{ Ethnicity } \\
\hline Dutch & $67.4 \%$ & $67.5 \%$ & $67.3 \%$ \\
\hline \multicolumn{4}{|l|}{ Child dietary assessments at 1 year } \\
\hline Age at dietary intake $(y)$ & $1.1(1.1-1.2)$ & $1.1(1.1-1.2)$ & $1.1(1.1-1.2)$ \\
\hline Total energy intake $(\mathrm{kcal} / \mathrm{d})$ & $1261(1060-1491)$ & $1210(1031-1438)$ & $1320(1095-1547)$ \\
\hline Diet quality at 1 year (score range $0-10$ ) & $4.3 \pm 1.3$ & $4.2 \pm 1.3$ & $4.4 \pm 1.3$ \\
\hline \multicolumn{4}{|l|}{ Breastfeeding in the first 4 months } \\
\hline Never & $9.3 \%$ & $8.9 \%$ & $9.8 \%$ \\
\hline Partially & $63.9 \%$ & $64.1 \%$ & $63.9 \%$ \\
\hline Exclusively & $26.7 \%$ & $27.0 \%$ & $26.4 \%$ \\
\hline \multicolumn{4}{|l|}{ Child dietary assessments at 8 years } \\
\hline Age at dietary intake $(\mathrm{y})$ & $8.1(8.0-8.2)$ & $8.1(8.0-8.2)$ & $8.1(8.0-8.2)$ \\
\hline Total energy intake (kcal/d) & $1461(1239-1703)$ & $1398(1191-1613)$ & $1537(1301-1770)$ \\
\hline Diet quality at 8 years (score range $0-10$ ) & $4.6 \pm 1.2$ & $4.5 \pm 1.2$ & $4.6 \pm 1.2$ \\
\hline \multicolumn{4}{|l|}{ Child growth measurements at 10 years } \\
\hline Age $(y)$ & $9.7(9.6-9.8)$ & $9.7(9.6-9.8)$ & $9.7(9.6-9.8)$ \\
\hline Height $(\mathrm{cm})$ & $141.4(137.2-145.8)$ & $141.2(136.8-145.8)$ & $141.5(137.4-145.8)$ \\
\hline Weight $(\mathrm{kg})$ & $33.6(30.2-38.0)$ & $33.6(30.0-38.2)$ & $33.6(30.4-37.8)$ \\
\hline Body mass index $\left(\mathrm{kg} / \mathrm{m}^{2}\right)$ & $16.8(15.6-18.3)$ & $16.8(15.5-18.5)$ & $16.7(15.7-18.2)$ \\
\hline Fat mass index $\left(\mathrm{kg} / \mathrm{m}^{2}\right)$ & $4.1(3.3-5.5)$ & $4.5(3.7-5.9)$ & $3.7(3.0-4.9)$ \\
\hline Fat-free mass index $\left(\mathrm{kg} / \mathrm{m}^{2}\right)$ & $12.5(11.8-13.2)$ & $12.1(11.6-12.8)$ & $12.8(12.2-13.5)$ \\
\hline Body fat percentage $(\%)$ & $25.2(21.1-30.6)$ & $27.4(23.7-32.5)$ & $22.6(19.0-27.7)$ \\
\hline Overweight or obese $\mathrm{a}^{\mathrm{a}}$ & $14.5 \%$ & $16.0 \%$ & $12.9 \%$ \\
\hline \multicolumn{4}{|l|}{ Screen time } \\
\hline$\geq 2$ h/day & $51.3 \%$ & $46.2 \%$ & $56.5 \%$ \\
\hline \multicolumn{4}{|l|}{ Sports } \\
\hline$\geq 2 \mathrm{~h} /$ week & $66.4 \%$ & $59.2 \%$ & $73.9 \%$ \\
\hline \multicolumn{4}{|l|}{ Parental characteristics during 10-year visit } \\
\hline Maternal age $(\mathrm{y})$ & $42.1(39.1-44.7)$ & $42.0(39.0-44.4)$ & $42.1(39.2-44.8)$ \\
\hline Maternal BMI $\left(\mathrm{kg} / \mathrm{m}^{2}\right)$ & $24.4(22.2-27.6)$ & $24.3(22.2-27.8)$ & $24.5(22.2-27.6)$ \\
\hline \multicolumn{4}{|l|}{ Maternal education } \\
\hline Higher & $63.1 \%$ & $63.4 \%$ & $62.9 \%$ \\
\hline \multicolumn{4}{|l|}{ Maternal smoking } \\
\hline Never & $53.8 \%$ & $53.7 \%$ & $54.0 \%$ \\
\hline Former & $32.9 \%$ & $32.7 \%$ & $33.0 \%$ \\
\hline Current & $13.3 \%$ & $13.6 \%$ & $13.0 \%$ \\
\hline \multicolumn{4}{|l|}{ Household income } \\
\hline$\geq 2800$ Euros/month & $69.5 \%$ & $70.5 \%$ & $68.4 \%$ \\
\hline
\end{tabular}

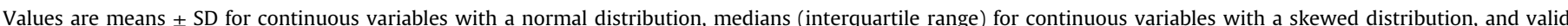
percentages for categorical variables. Missing data of covariates (ranging from $0 \%$ to $28.1 \%$ ) were imputed with multiple imputation ( $\mathrm{n}=10$ imputations).

a According to international age- and sex-specific cut-offs for BMI [19].

Table 2

Associations of diet quality at age 1 year with child's trajectories of growth and body composition up to the age of 10 years.

\begin{tabular}{|c|c|c|c|c|c|c|}
\hline & $\begin{array}{l}\text { Height } \\
(\mathrm{SDS}) n=3573\end{array}$ & $\begin{array}{l}\text { Weight } \\
(\mathrm{SDS}) n=3573\end{array}$ & $\begin{array}{l}\text { Body mass index } \\
\text { (SDS) } n=3573\end{array}$ & $\begin{array}{l}\text { Fat mass index } \\
(\mathrm{SDS}) n=3112\end{array}$ & $\begin{array}{l}\text { Fat-free mass } \\
\text { index (SDS) } n=3112\end{array}$ & $\begin{array}{l}\text { Percentage body fat } \\
\text { (SDS) } n=3112\end{array}$ \\
\hline \multicolumn{7}{|c|}{ Diet quality score 1 year } \\
\hline Model 1 (basic) & $0.05(0.02,0.08)$ & $0.05(0.02,0.08)$ & $0.04(0.01,0.06)$ & $-0.01(-0.04,0.02)$ & $0.03(0.002,0.06)$ & $-0.02(-0.05,0.01)$ \\
\hline Model 2 (confounder) & $0.05(0.02,0.08)$ & $0.06(0.04,0.09)$ & $0.05(0.02,0.08)$ & $0.02(-0.01,0.05)$ & $0.04(0.004,0.07)$ & $0.01(-0.02,0.04)$ \\
\hline Model 3 (DQ 8 y) & $0.04(0.01,0.07)$ & $0.06(0.03,0.09)$ & $0.05(0.02,0.07)$ & $0.02(-0.01,0.05)$ & $0.03(-0.01,0.06)$ & $0.01(-0.02,0.04)$ \\
\hline
\end{tabular}

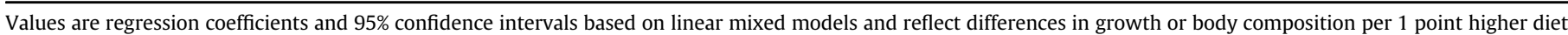
quality score. Bold values indicate statistically significant effect estimates.

Model 1 (basic): adjusted for gender, ethnicity, age dietary assessment, and total energy intake.

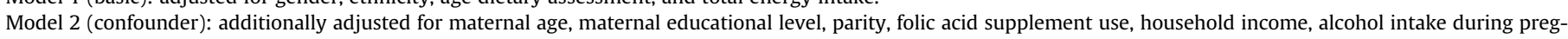
nancy, smoking during pregnancy, breastfeeding, playing sports, and screen time.

Model 3 (diet quality $8 \mathrm{y}$ ): additionally adjusted for diet quality at the age of 8 years.

after additional adjustment for infant diet quality $(\beta=0.06,95 \% \mathrm{Cl}$ : 0.04, 0.09) (model 3, Table 3).

After stratification by sex (p-for-interaction $<0.05$ for height, weight, and BMI), associations for diet quality at 8 years with anthropometrics only remained in girls, but not in boys (Table 4 ). The positive association of diet quality at age 8 years with FFMI at age 10 years was observed for both boys and girls, but the effect estimate was larger in girls than in boys ( $\beta=0.09$ SDS, $95 \% \mathrm{CI}$ : $0.05,0.12$ in girls versus $\beta=0.04,95 \% \mathrm{CI}$ : $0.000,0.07$ in boys) (model 3, Table 4).

\subsection{Sensitivity analyses}

Interactions of diet quality with age at outcome measurements were not statistically significant. This suggests that diet quality 
Table 3

Associations of diet quality at age 8 years with child's growth and body composition at the age of 10 years.

\begin{tabular}{|c|c|c|c|c|c|c|}
\hline & $\begin{array}{l}\text { Height (SDS) } \\
n=3991\end{array}$ & $\begin{array}{l}\text { Weight } \\
(\mathrm{SDS}) n=3991\end{array}$ & $\begin{array}{l}\text { Body mass index } \\
\text { (SDS) } n=3991\end{array}$ & $\begin{array}{l}\text { Fat mass index } \\
(\mathrm{SDS}) n=3950\end{array}$ & $\begin{array}{l}\text { Fat-free mass index } \\
(\mathrm{SDS}) n=3950\end{array}$ & $\begin{array}{l}\text { Percentage body fat } \\
(\mathrm{SDS}) n=3950\end{array}$ \\
\hline \multicolumn{7}{|c|}{ Diet quality score 8 years } \\
\hline Model 1 (basic) & $0.06(0.04,0.09)$ & $0.01(-0.01,0.03)$ & $-0.02(-0.04,0.004)$ & $-0.05(-0.07,-0.03)$ & $0.06(0.03,0.08)$ & $-0.07(-0.09,-0.04)$ \\
\hline Model 2 (confounder) & $0.06(0.03,0.08)$ & $0.04(0.02,0.07)$ & $0.03(0.003,0.05)$ & $0.001(-0.02,0.02)$ & $0.07(0.05,0.10)$ & $-0.01(-0.04,0.01)$ \\
\hline Model 3 (DQ 1 y) & $0.05(0.02,0.08)$ & $0.04(0.01,0.06)$ & $0.02(-0.003,0.04)$ & $-0.001(-0.03,0.02)$ & $0.06(0.04,0.09)$ & $-0.02(-0.04,0.01)$ \\
\hline
\end{tabular}

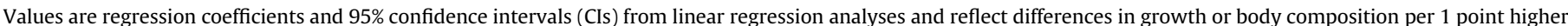
diet quality score. Bold values indicate statistically significant effect estimates.

Model 1 (basic): adjusted for gender, ethnicity, age dietary assessment, and total energy intake.

Model 2 (confounder): additionally adjusted for maternal educational level, playing sports, screen time, maternal smoking, household income, and maternal BMI.

Model 3 (diet quality $1 \mathrm{y}$ ): additionally adjusted for diet quality at age 1 year and breastfeeding.

Table 4

Associations of diet quality score at 8 years with child's growth and body composition at the age of 10 years stratified for sex.

\begin{tabular}{|c|c|c|c|c|c|c|}
\hline & Height (SDS) & Weight (SDS) & Body mass index (SDS) & Fat mass index (SDS) & Fat-free mass index (SDS) & Percentage body fat (SDS) \\
\hline Girls & $n=2022$ & $n=2022$ & $n=2022$ & $n=2004$ & $n=2004$ & $n=2004$ \\
\hline \multicolumn{7}{|c|}{ Diet quality score 8 years } \\
\hline Model 1 (basic) & $0.09(0.06,0.13)$ & $0.04(0.01,0.08)$ & $0.01(-0.03,0.04)$ & $-0.03(-0.06,0.001)$ & $0.08(0.04,0.11)$ & $-0.06(-0.09,-0.02)$ \\
\hline Model 2 (confounder) & $0.09(0.05,0.12)$ & $0.07(0.04,0.10)$ & $0.05(0.01,0.08)$ & $0.01(-0.02,0.04)$ & $0.10(0.06,0.13)$ & $-0.01(-0.05,0.02)$ \\
\hline Model 3 (DQ 1 y) & $0.07(0.03,0.11)$ & $0.06(0.02,0.09)$ & $0.04(0.004,0.07)$ & $0.004(-0.03,0.04)$ & $0.09(0.05,0.12)$ & $-0.02(-0.05,0.02)$ \\
\hline Boys & $n=1969$ & $n=1969$ & $n=1969$ & $n=1946$ & $n=1946$ & $n=1946$ \\
\hline \multicolumn{7}{|c|}{ Diet quality score 8 years } \\
\hline Model 1 (basic) & $0.03(-0.003,0.07)$ & $-0.02(-0.06,0.01)$ & $-0.04(-0.08,-0.01)$ & $-0.07(-0.10,-0.03)$ & $0.03(-0.003,0.07)$ & $-0.08(-0.11,-0.04)$ \\
\hline Model 2 (confounder) & $0.03(-0.01,0.07)$ & $0.02(-0.02,0.05)$ & $0.01(-0.03,0.04)$ & $-0.01(-0.04,0.03)$ & $0.04(0.01,0.08)$ & $-0.01(-0.05,0.02)$ \\
\hline Model 3 (DQ 1 y) & $0.03(-0.01,0.06)$ & $0.01(-0.02,0.05)$ & $0.002(-0.03,0.04)$ & $-0.01(-0.04,0.03)$ & $0.04(0.000,0.07)$ & $-0.01(-0.05,0.02)$ \\
\hline
\end{tabular}

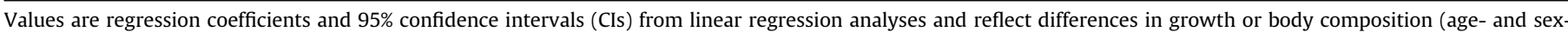

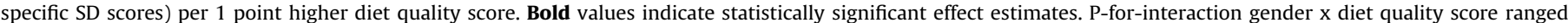
from 0.01 to 0.04 for growth and from 0.14 to 0.58 for body composition.

Model 1 (basic): adjusted for ethnicity, age dietary assessment, and total energy intake.

Model 2 (confounder): additionally adjusted for maternal educational level, playing sports, screen time, maternal smoking, household income, and maternal BMI.

Model 3 (diet quality $1 \mathrm{y}$ ): additionally adjusted for diet quality at age 1 year and breastfeeding.

does not affect the velocity of growth or body composition. Analyses restricted to children with a Dutch ethnic background (n between 2145 and 2691) yielded similar effect estimates as compared to the whole group (Supplemental Tables 1 and 2). In this subgroup, associations of diet quality at age 1 year with FFMI up to age 10 years remained statistically significant also in model 3 . Analyses in which we excluded outcome measurements that were taken during the first year following dietary assessment showed similar associations as in the main models, suggesting that body size around the time of food intake assessment does not seem to drive our findings (Supplemental Table 3). Sensitivity analyses with diet quality scores excluding one component stepwise at a time and analyses excluding children with overweight or obesity at baseline also showed similar effect estimates (data not shown).

\section{Discussion}

In this population-based cohort study, we observed that better diet quality, both in early and mid-childhood, was associated with higher height and weight up to the age of 10 years, independent of diet quality at the other time point. The association of diet quality with higher weight was explained by a higher fat-free mass, and not fat mass or BF\%. For diet quality in mid-childhood, effect estimates were generally higher in girls compared to boys.

\subsection{Interpretation and comparison with previous studies}

In line with our previous findings that higher diet quality in early childhood is associated with higher height, weight, BMI, and FFMI at age 6 years in the Generation R Study [13], our current findings show that these associations remain up to age 10 years. In addition, we observed that most of these associations were independent of diet quality in later childhood, which emphasizes the importance of early-childhood diet on growth and body composition. Also for mid-childhood diet, these associations were independent of diet in early childhood, suggesting that not only early-childhood diet is of high importance, but that dietary intake in later childhood is important as well. Overall diet quality in our population was suboptimal and not conform age-specific dietary guidelines $[15,16]$, but in line with other studies on diet quality of children in Western countries [21,22]. Although previous studies suggested that diet quality may track throughout childhood [23], diet quality at the young age of 1 year and at age 8 years in our study population was only weakly correlated [16]. Although the two diet quality scores that we used were not exactly the same (e.g. a few differences in food groups and different cut off values), these differences reflect differences in age-specific dietary guidelines. Both scores thus reflect level of adherence to dietary guidelines for that age. Our findings that both diet quality in early and midchildhood are important emphasize that children should have a healthy diet in early childhood, but should also maintain this healthy diet throughout childhood for optimal growth and to prevent the development of obesity.

For diet quality at age 8 years, associations were stronger in girls than boys. Diet quality did not differ between boys and girls at either time point $[15,16]$. Given the age of our study population of 10 years at the final body composition assessment, children may be at different peri-pubertal stages. As puberty starts at an earlier age in girls than boys, developmental changes associated with puberty, such as the growth spurt and hormonal changes, may explain the stronger associations of diet quality with growth and body composition among girls. The analyses of diet quality in midchildhood may support this as only body composition measurements at age 10 years were included in these analyses. As suggested 
by Wells et al., height should be taken into account in measurements of body composition, especially during this stage of child's development in which rapid growth occurs [24]. The importance of height in associations of diet with body composition in children is also supported by findings from a Canadian study, which showed that better diet quality was associated with lower BF\% in children aged 8-10 years, but not with BMI or FMI, in which height is taken into account [12]. Unfortunately, sex differences in these associations were not examined. In addition to the difference in timing of growth spurt between boys and girls, hormonal changes that occur during puberty can also influence body composition differently; from onset of puberty onwards, the percentage of body fat is generally higher in girls than boys [25]. Indeed, also in our study population, girls had a higher FMI and a lower FFMI than boys. Further study is needed to examine sex differences in the associations of diet in childhood with growth and body composition at different ages and to study whether these differences track into adolescence and adulthood.

Previously, researchers from the ALSPAC Study in the UK used reduced rank regression to identify a data-driven energy-dense, high-fat, low-fiber dietary pattern at children's ages of 7,10, and 13 years. This pattern was associated with a higher FMI at the ages of 11,13 , and 15 years [11]. Other studies reported a lower weight, BMI, or BF\% among children with a healthier dietary pattern $[12,13,22]$. Given this previous evidence, we had expected that children with a higher diet quality would have a lower weight and FMI, but instead we observed associations with a higher weight and FFMI. These partly contrasting findings could be explained by the use of different dietary patterns. One of the previously described studies in British children used a diet quality index [22]. This diet quality index included intakes of both food groups and nutrients (including fruit, vegetables, bread and cereals, but also total fat, saturated fat, cholesterol, protein, sodium, and calcium). Contrary, our diet quality score included only intakes of food groups, which may make associations difficult to compare. Also, in our diet quality score, healthier and less healthy choices were taken into account within the components. For example, we included healthy fats (i.e., vegetable oils and soft margarine) rather than total fat, and we included whole-grain products rather than total grains. Indeed, for both the early and mid-childhood diet scores in our study, good construct validity for nutrient intakes was observed $[15,16]$. In addition, the diet quality index used a categorical scoring system; for each component of the diet quality index, participants could score 0,1 , or 2 points, whereas our scoring system was continuous, thereby providing better discrimination [26]. Since their diet quality index and our diet quality score were constructed differently, these scores could represent different dietary patterns, which may explain why the British study observed a lower weight and BMI in children with a higher diet quality, whereas we observed a higher weight and BMI among those with a healthier diet. However, the overall health effect may be similar, as our associations with higher BMI were fully driven by a higher FFMI, and not FMI. Therefore, evidence from both this previous study and our current study suggests that a healthy dietary pattern may prevent the development of adiposity in children, through a lower fat mass and/ or a higher fat-free mass.

\subsection{Strengths and limitations}

Strengths of this study include its large sample size, the population-based, longitudinal design, and the availability of data on several potential confounders. Another important strength is that measures of body composition were assessed with DXA-scans, allowing us to distinguish between fat mass and fat-free mass, since BMI only is not an adequate measure of adiposity $[27,28]$. A few previous studies used skinfold thickness to estimate adiposity, but this method has been shown to underestimate body fat in children [29]. Therefore, especially among growing children, it is important to study the role of diet in obesity using accurate and detailed measures of body composition, assessed with for example DXAscans. Furthermore, we evaluated overall dietary intake rather than single nutrients or food products. Following this approach, we were able to take into account the high interactions between nutrients and foods within a diet [9]. In addition, we had data on dietary intake available at two different moments during childhood, one as a measure for early-childhood diet and one for midchildhood diet, and both diet quality scores have been shown to have good construct validity $[15,16]$. This allowed us to study whether associations of diet with anthropometrics and body composition were independent of diet at an earlier or later time point in childhood, However, dietary intake data at more time points throughout childhood would have been better to perform longitudinal analyses.

Several limitations should be taken into account as well. Dietary intake was assessed with FFQs, which may be subject to measurement errors [30]. However, FFQs have shown to be able to accurately rank participants according to their dietary intake [30]. In addition, results from validation studies using the doubly labeled water method [18] or against repeated $24 \mathrm{~h}$ recalls [15] showed moderate to good validity of the FFQs used in our study. Although both FFQs were originally developed for and validated in Dutch children and our study population has a multi-ethnic background, sensitivity analyses restricted to children with a Dutch ethnic background showed similar results, suggesting no large bias due to ethnicity. Although we were able to control for several socioeconomic and lifestyle factors, some of these factors may not have been measured perfectly and we could have missed some important factors. For example, we did not have information on pubertal status and no detailed information on physical activity. For the latter, we used amount of time playing sports as a proxy, which could have led to residual confounding. Finally, most of the participants included in our study had a Dutch ethnic background, were highly educated, and had a high household income, which may limit the generalizability of our findings to other populations.

\section{Conclusion}

In conclusion, we observed that higher diet quality, both in early and mid-childhood, was associated with higher height, weight, and FFMI up to the age of 10 years, independent of diet at the other time point. Our findings suggest that a healthy diet according to dietary guidelines, during several stages of childhood, has a beneficial effect on growth and may decrease the risk of adiposity.

\section{Funding sources}

The Generation R Study is made possible by financial support from Erasmus Medical Center (EMC), Rotterdam, Erasmus University Rotterdam (EUR), and the Netherlands Organization for Health Research and Development (ZonMw) 'Geestkracht' program (10.000.1003). VWVJ received an additional grant from the Netherlands Organization for Health Research and Development (ZonMw VIDI: 016.136.361). The funders were not involved in the study design; collection, analysis, and interpretation of the data; writing of the report; or in the decision to submit this article for publication.

\section{Conflicts of interest}

None of the authors declares a financial or personal conflict of interest related to this work. 


\section{Acknowledgements}

The Generation R Study is conducted by Erasmus Medical Center in close collaboration with the School of Law and the Faculty of Social Sciences at the Erasmus University, Rotterdam; the Municipal Health Service, Rotterdam area; and the Stichting Trombosedienst \& Artsenlaboratorium Rijnmond (Star-MDC), Rotterdam. We gratefully acknowledge the contribution of participating mothers, general practitioners, hospitals, midwives, and pharmacies in Rotterdam, the Netherlands. The authors' responsibilities were as follows: ANN and TV designed the research project; ANN, VJ, and TV analyzed the data; VWVJ, FR, and MAI were involved in the study design and data collection; VWVJ, FR, PWJ, and MAI provided consultation regarding the analyses and interpretation of the data; ANN and TV wrote the paper and had primary responsibility for final content. All authors read and approved the final manuscript.

\section{Appendix A. Supplementary data}

Supplementary data to this article can be found online at https://doi.org/10.1016/j.clnu.2019.03.017.

\section{References}

[1] Langley-Evans SC. Nutrition in early life and the programming of adult disease: a review. J Hum Nutr Diet 2015;28(s1):1-14.

[2] Ludwig DS, Peterson KE, Gortmaker SL. Relation between consumption of sugar-sweetened drinks and childhood obesity: a prospective, observational analysis. Lancet 2001;357(9255):505-8.

[3] Braun KV, Erler NS, Kiefte-de Jong JC, Jaddoe VW, van den Hooven EH, Franco $\mathrm{OH}$, et al. Dietary intake of protein in early childhood is associated with growth trajectories between 1 and 9 Years of age. J Nutr 2016;146(11): 2361-7.

[4] Tucker LA, Seljaas GT, Hager RL. Body fat percentage of children varies according to their diet composition. J Am Diet Assoc 1997;97(9):981-6.

[5] Rolland-Cachera MF, Deheeger M, Akrout M, Bellisle F. Influence of macronutrients on adiposity development: a follow up study of nutrition and growth from 10 months to 8 years of age. Int J Obes Relat Metab Disord - J Int Assoc Stud Obes 1995;19(8):573-8.

[6] Voortman T, Braun KVE, Kiefte-de Jong JC, Jaddoe VWV, Franco OH, van den Hooven EH. Protein intake in early childhood and body composition at the age of 6 years: the Generation R Study. Int J Obes 2016;40(6):1018-25.

[7] Singh AS, Mulder C, Twisk JWR, Van Mechelen W, Chinapaw MJM. Tracking of childhood overweight into adulthood: a systematic review of the literature. Obes Rev 2008;9(5):474-88.

[8] Ebbeling CB, Pawlak DB, Ludwig DS. Childhood obesity: public-health crisis, common sense cure. Lancet 2002;360(9331):473-82.

[9] Hu FB. Dietary pattern analysis: a new direction in nutritional epidemiology. Curr Opin Lipidol 2002;13(1):3-9.

[10] Ambrosini GL. Childhood dietary patterns and later obesity: a review of the evidence. Proc Nutr Soc 2014;73(01):137-46.
[11] Ambrosini GL, Emmett PM, Northstone K, Howe LD, Tilling K, Jebb SA. Identification of a dietary pattern prospectively associated with increased adiposity during childhood and adolescence. Int J Obes 2012;36(10): 1299-305.

[12] Setayeshgar S, Maximova K, Ekwaru JP, Gray-Donald K, Henderson M, Paradis G, et al. Diet quality as measured by the Diet Quality IndexInternational is associated with prospective changes in body fat among Canadian children. Publ Health Nutr 2017;20(3):456-63.

[13] Voortman T, Leermakers ETM, Franco OH, Jaddoe VWV, Moll HA, Hofman A et al. A priori and a posteriori dietary patterns at the age of 1 year and body composition at the age of 6 years: the Generation R Study. Eur J Epidemiol 2016:1-9.

[14] Kooijman MN, Kruithof CJ, van Duijn CM, Duijts L, Franco OH, van IJzendoorn MH, et al. The Generation R Study: design and cohort update 2017. Eur J Epidemiol 2016;31(12):1243-64.

[15] Voortman T, Kiefte-de Jong JC, Geelen A, Villamor E, Moll HA, de Jongste JC, et al. The development of a diet quality score for preschool children and its validation and determinants in the Generation R Study. J Nutr 2015;145(2): 306-14.

[16] van der Velde LA, Nguyen AN, Schoufour JD, Geelen A, Jaddoe VWV, Franco $\mathrm{OH}$, et al. Diet quality in childhood: the generation R study. Eur J Nutr 2018. https://doi.org/10.1007/s00394-018-1651-z.

[17] Kiefte-de Jong JC, de Vries JH, Bleeker SE, Jaddoe VW, Hofman A, Raat H, et al. Socio-demographic and lifestyle determinants of 'Western-like' and 'Health conscious' dietary patterns in toddlers. Br J Nutr 2013;109(1):137-47.

[18] Dutman AE, Stafleu A, Kruizinga A, Brants HAM, Westerterp KR, Kistemaker C, et al. Validation of an FFQ and options for data processing using the doubly labelled water method in children. Publ Health Nutr 2011;14(03):410-7.

[19] Cole TJ, Bellizzi MC, Flegal KM, Dietz WH. Establishing a standard definition for child overweight and obesity worldwide: international survey. BM] 2000;320(7244):1240-3.

[20] Rubin DB, Schenker N. Multiple imputation in health-are databases: an overview and some applications. Stat Med 1991;10(4):585-98.

[21] Carlson A, Lino M, Gerrior S, Basiotis PP. Insight 25: September 2001: report card on the diet quality of children ages 2 to 9 . Fam Econ Nutr Rev $2003 ; 15(2)$ : $52-5$.

[22] Jennings A, Welch A, van Sluijs EMF, Griffin SJ, Cassidy A. Diet quality is independently associated with weight status in children aged 9-10 years. J Nutr 2011;141(3):453-9.

[23] Northstone K, Emmett PM. Are dietary patterns stable throughout early and mid-childhood? A birth cohort study. Br J Nutr 2008;100(05):1069-76.

[24] Wells JCK, Cole TJ. Adjustment of fat-free mass and fat mass for height in children aged 8 y. Int J Obes 2002;26(7):947.

[25] Loomba-Albrecht LA, Styne DM. Effect of puberty on body composition. Curr Opin Endocrinol Diabetes Obes 2009;16(1):10-5.

[26] Waijers PMCM, Feskens EJM, Ocké MC. A critical review of predefined diet quality scores. Br J Nutr 2007;97(02):219-31.

[27] Wells JC. A Hattori chart analysis of body mass index in infants and children. Int J Obes 2000;24(3):325-9.

[28] Freedman DS, Wang J, Maynard LM, Thornton JC, Mei Z, Pierson RN, et al Relation of BMI to fat and fat-free mass among children and adolescents. Int J Obes 2005;29(1):1-8.

[29] Eisenmann JC, Heelan KA, Welk GJ. Assessing body composition among 3-to 8year-old children: anthropometry, bia, and dxa. Obes Res 2004;12(10): $1633-40$.

[30] Kipnis V, Subar AF, Midthune D, Freedman LS, Ballard-Barbash R, Troiano RP, et al. Structure of dietary measurement error: results of the OPEN biomarker study. Am J Epidemiol 2003;158(1):14-21. 